\title{
Filigrane
}

Écoutes psychothérapiques

\section{La clinique des expériences limites. Entrevue avec Odette Arsenault}

\section{Thérèse Nadeau}

Volume 16, numéro 1, printemps 2007

Les hauts lieux et non-lieux du rêve I

URI : https://id.erudit.org/iderudit/016179ar

DOI : https://doi.org/10.7202/016179ar

Aller au sommaire du numéro

Éditeur(s)

Revue Santé mentale au Québec

ISSN

1192-1412 (imprimé)

1911-4656 (numérique)

Découvrir la revue

Citer cet article

Nadeau, T. (2007). La clinique des expériences limites. Entrevue avec Odette Arsenault. Filigrane, 16(1), 85-98. https://doi.org/10.7202/016179ar d'utilisation que vous pouvez consulter en ligne.

https://apropos.erudit.org/fr/usagers/politique-dutilisation/ 


\title{
La clinique des expériences limites. Entrevue avec Odette Arsenault
}

\author{
thérèse nadeau
}

T.N. : Merci, Madame Arsenault, d'avoir accepté cette entrevue pour la revue Filigrane. Vous avez travaillé pendant presque 20 ans, de 1983 à 2002, en intervention individuelle et de groupe auprès de gens victimes d'incidents traumatiques. Nous souhaiterions entendre vos réflexions sur ce champ clinique. Peutêtre pourriez-vous d'abord nous dire comment vous en êtes arrivée à travailler en post-traumatique.

O.A.: C'est un hasard qui m'a amenée au post-traumatique. À l'époque, au début des années 1980, j'avais déjà une dizaine d'années d'expérience en tant que psychologue. J'avais été formée à l'intervention de crise, à la dynamique des groupes, au counseling individuel et je venais d'accepter de m'occuper des programmes d'aide aux employés (PAE) de la Fédération des Caisses populaires Desjardins de Montréal et de l'Ouest du Québec. Les PAE étaient naissants au Québec à cette époque et offraient des consultations individuelles à court terme pour les employés. Or, dans les Caisses populaires surviennent périodiquement des vols à main armée. La Sécurité Desjardins - maintenant l'organisme porte un autre nom - a donc communiqué avec moi: «Madame Arsenault, vous vous occupez du programme d'aide aux employés. Vous soignez tous les problèmes des employés, les problèmes de couples, les états dépressifs, l'alcoolisme, mais vous ne faites rien pour les victimes de hold-up. Nous, nous allons dans les Caisses après un vol à main armée et on sait que les filles ont de la misère après les hold-up »... C'est ainsi que j'ai commencé à m'intéresser à ce champ d'intervention psychologique.

À Montréal, à l'époque, seules une ou deux personnes s'occupaient de cette problématique. J.T. Mitchell avait commencé à faire de l'intervention aux ÉtatsUnis. Il donnait des séances de formation ici et là. J'ai donc commencé à lire, à m'inscrire à des ateliers de formation et à intervenir - au début presque exclusivement — dans les Caisses populaires Desjardins. Et j'ai pris de l'expérience. Au début je m'adressais aux victimes en disant: «Vous venez de vivre un événement difficile, en dehors des stress habituels. Si vous voulez, on va en parler et essayer de comprendre ce qui vous arrive, comment je peux vous aider à passer à travers une telle épreuve et ce que vous devez éviter pour ne pas vous nuire. » J'y allais de façon très prudente. Je ne voulais pas bousculer les gens Les victimes m'ont beaucoup appris en parlant spontanément de leur expérience, parce qu'elles en 
avaient grand besoin : «Qu'est-ce qui nous arrive ? Qu'est-ce qu'on est en train de devenir? Est-ce que ça va durer toujours?

T.N. : Parce que les gens ont peur en constatant leurs réactions tant physiologiques qu'émotionnelles ?

O.A.: Oui. Je dirais que l'essentiel de l'intervention post-traumatique de groupe, ou du début d'une psychothérapie avec des victimes de traumatismes ponctuels, l'essentiel de l'intervention consiste à alléger l'anxiété entourant les réactions émotionnelles intenses qui surgissent inévitablement. Les victimes de traumatisme subissent sans avertissement, une intensité émotionnelle beaucoup plus grande que celle à laquelle elles sont habituées. Non seulement la nature des émotions mais leur intensité est traumatique. Nous parlons ici de traumatismes ponctuels et non de traumatismes cumulatifs ou de violence intra-familiale où la dynamique est plus complexe. Mon propos concerne surtout les accidents mortels ou les actes criminels sur les lieux de travail, dans les entreprises ou les institutions, les catastrophes naturelles etc. Les victimes sont donc des gens fonctionnels. Les agressions ou les accidents surviennent subitement. Les gens ne sont pas préparés. Ils sont surpris, en plus d'être terrorisés. Une des premières choses à faire consiste donc à les aider à tolérer puis à apprivoiser ce qui se passe dans leur vie intérieure... et pour quelques-uns c'est la première fois qu'ils découvrent qu'ils ont une vie intérieure.

T.N.: Oui, c'est ça.

O.A.: L'intervention post-traumatique de groupe comprend un aspect psychopédagogique. D'abord aider les victimes à comprendre que ce qu'elles vivent est normal dans les circonstances. Ce sont les circonstances qui sont anormales, et non leurs réactions. Et, dans un premier temps, toute réaction est considérée comme normale, y compris l'absence de réaction.

T.N.: Dans les années 1980, le DSM (Diagnostic and Statistical Manual of Mental Disorders de l'American Psychiatric Association) commençait à introduire le concept de stress post-traumatique. Mais dans les grandes institutions, il n'y avait pas de professionnels formés pour ce type de problèmes, n'est-ce pas?

O.A. : Non. Le DSM était utilisé dans les hôpitaux, mais pas par les psychologues travaillant en pratique privée, dans les entreprises ou les commissions scolaires. Au début des années 1980 on ne se servait pas encore du DSM concernant le stress post-traumatique

T.N.: Ce n'était pas un trouble bien identifié dans ses particularités, dans son expression?

O.A.: Pas à ce moment-là. Maintenant, le DSM donne trois définitions de l'état de stress post-traumatique: le stress aigu dans les semaines qui suivent, le stress chronique qui dure plus de trois mois après l'événement et le stress en survenue différée qui peut apparaître plus de six mois après l'événement. Je pense qu'il faut s'occuper des gens en stress aigu, les supporter, faire de la prévention, afin que ce stress premier ne se chronicise pas.

T.N. : Non. 
O.A.: Si les réactions liées au stress s'atténuent à l'intérieur de trois mois, elles constituent, selon moi, des réactions normales à une situation anormale. Si elles ne se résorbent pas, si un cheminement ne s'effectue pas, on se trouve devant une psychopathologie et il faut alors travailler avec cette réalité. Mais dans un premier temps, il est normal de manifester des défenses plus fortes, de réagir par une intensité émotionnelle et une détresse subjective plus grandes qu'à l'habitude.

T.N. : Alors, comment identifier un événement traumatique? Comment est-ce qu'on pourrait le définir ou le représenter? Vous avez parlé, par exemple, d'un hold-up dans une banque, vous avez fait référence à Polytechnique. On peut imaginer des tremblements de terre... Vous avez dit: «Je ne parlerai pas des stress cumulatifs vécus dans le secret des familles, qui sont aussi des traumatismes mais d'un autre ordre. Je parlerai du traumatisme qui comme un coup de tonnerre...

O.A. : C'est comme un coup de tonnerre, sans avertissement, tout à fait.

T.N. : Alors comment vous définissez-vous un événement traumatique?

O.A. : Le DSM dit que la personne doit être exposée — comme victime ou comme témoin - à une situation impliquant un danger, une menace réelle ou potentielle à la vie ou à l'intégrité physique. C'est un événement qui, tel que vécu par la personne, implique une menace de mort. En général, ce sont des événements publics mais ce n'est pas la description de l'événement qui détermine si la personne a vécu un stress traumatique, c'est ce qu'elle a vécu elle-même: des sentiments de terreur, d'horreur, d'impuissance. Par exemple, un voleur arrive avec un fusil dans une Caisse Populaire ou une banque et dit: «Haut les mains!» Les gens sont pris par surprise et terrorisés, ils ont peur qu'il tire. Par la suite, dans l'après-coup, si les employés apprennent que le fusil n'était pas chargé, ils ont beaucoup de difficulté à comprendre que cette information ne change en rien leur vécu pendant le hold-up. Souvent malheureusement, une nouvelle information comme celle-là va changer la perception de l'entourage envers les victimes et accroître ainsi leur sentiment d'incompréhension et de solitude. C'est la perception subjective des événements qui est déterminante et non la découverte de faits nouveaux dans l'après-coup. Le voleur, tel que perçu par les victimes, menaçait leur vie, même s'il utilisait un fusil non chargé. Cet aspect est extrêmement important. À Polytechnique, en décembre 1989 , les gens ont dit aux victimes masculines : «Mais le tueur visait seulement les filles, pourquoi vous, les hommes, avez-vous eu peur et pourquoi n'avez-vous pas défendu les filles?»C'est que, pendant l'événement, ces étudiants ne savaient pas que le tueur ne visait que les étudiantes. Cette information fut une constatation d'après coup. Pendant l'événement, il n'y avait que des victimes qui entendaient les balles passer, qui voyaient les gens tomber et qui avaient peur pour leur vie. Et tout ça se fait dans un climat de désorganisation.

T.N.: Oui, le drame avait un effet de sidération. La pensée était mise totalement hors circuit.

O.A. : C'est ça. Les gens sont dans l'horreur ou dans la terreur; ils éprouvent un sentiment d'impuissance absolument total, ils sont dans une situation de très grande angoisse, de très grande menace. 
T.N.: J'imagine qu'au moment où les événements se produisent, les notions d'espacetemps, de durée, d'orientation, deviennent confuses. Un arrêt de la pensée et de la capacité de se représenter le déroulement des événements eux-mêmes doit survenir.

O.A.: Oui, certainement. La première étape d'une réaction traumatique consiste en un état de choc qui peut durer quelques heures ou quelques jours (tel que décrit par M.J. Horowitz). Mais pendant l'événement lui-même, les gens ne peuvent pas penser et ils ne peuvent même pas ressentir clairement des émotions. Les gens sont pétrifiés dans l'horreur ou dans la terreur. On constate des distorsions dans la perception de la durée du temps. Les gens diront: «Mais le hold-up a duré trente minutes...». Alors que cela a duré, en fait, une minute et demie. Les gens sont en survie.

J'ai toujours eu beaucoup d'admiration pour ceux et celles qui vivent ce genre d'épreuve. Ils font de leur mieux dans les circonstances et c'est rare que quelqu'un aggrave, par son comportement, le danger de la situation. L'instinct de survie les guide. Les gens semblent savoir jusqu' où ils peuvent aller, ce qu'ils peuvent dire, ne pas dire à l'agresseur. En général, ils réagissent avec une grande maîtrise pendant l'événement. Ce n'est qu'après que se manifestent les émotions. Mais pendant l'agression, il n'y a pas de réflexion. Pendant l'événement, certains peuvent éprouver la peur de mourir; d'autres, non, ils ne sont que sidérés. Plusieurs vivent quelques heures ou quelques jours de dissociation: «Je parle, c'est comme si je parlais à travers un nuage»; «tout me semble plus lointain que d'habitude»; «Je ne suis pas capable de saisir les choses comme avant».

T.N.: Oui. Cela doit faire partie des symptômes angoissants.

O.A.: Tout à fait. Je suis d'avis qu'il faut expliquer aux victimes que leurs symptômes sont la conséquence d'une trop grande souffrance morale ou d'une protection contre celle-ci. Les gens ont besoin de comprendre que cette émotion, cette sensation cette difficulté de concentration ont une raison d'être. Une émotion comporte une dimension physiologique; elle n'existe pas seulement dans l'esprit. Le corps réagit, les hormones s'ajustent. Des recherches ont mesuré les réactions physiologiques de l'organisme après un traumatisme. L'hyper-vigilance, les flashbacks, les sursauts, tout ce que les victimes éprouvent s'explique physiologiquement. Le corps reste pris en tension de survie.

T.N. : En tension de survie qui préparait à la lutte...

O.A.: À la survie physique et à la préservation de l'équilibre psychique. Les efforts furent tellement grands que la tension prend du temps à se résorber par la suite. La compréhension de ces phénomènes physiologiques augmente la tolérance à une souffrance normale dans les circonstances. Sinon, cet état serait vu comme trop proche de la folie. Et la souffrance morale comme la folie font peur, alors... T.N.: Oui. L'intervention qu'on appelle le debriefing ne s'effectue pas tout de suite, dans ces cas. On doit d'abord mettre les gens en situation de sécurité, les sortir de l'endroit où l'événement s'est déroulé. L'intervention aura lieu dans les heures ou les journées qui suivent.

O.A.: Je suis tout à fait d'accord. Les recherches montrent que les interventions psychologiques font une différence sur la suite des choses et que plus elles 
surviennent rapidement, mieux les victimes s'en portent. Mais il faut s'entendre, cette rapidité ne signifie pas une intervention immédiate. Selon moi, le jour même, il faut faire place à la compassion, faire venir les conjoints, préparer du café, ce sont là des gestes humains essentiels qui ne sont pas de l'ordre de l'acte professionnel. D'après mon expérience, l'intervention psychologique proprement dite ne doit jamais avoir lieu le jour même. Tous les praticiens ne sont pas de cet avis. Mais je pense qu'elle doit avoir lieu le lendemain et même quelques jours plus tard, pour laisser aux gens le temps de sentir un mouvement dans leur vie intérieure, de passer d'un état de choc à l'apparition de l'intensité émotionnelle, qui constitue la deuxième étape de la réaction traumatique, la première étant l'état de choc, la sidération.

Lors des bouleversements dramatiques et subits, la plus grande difficulté pour les victimes réside dans la perte de contrôle de leur vie intérieure. Normalement, on est en mesure d'exercer un certain contrôle sur cette dernière: si on est tendu, on peut faire quelque chose pour se détendre, si on est en conflit avec quelqu'un, on peut en parler ou agir. Tout d'un coup, subitement, un événement survient et les victimes sont aux prises avec une peur continuelle, avec des cauchemars, des flashbacks...

T.N.: C'est qu'ils n'étaient pas préparés à la survenue d'un événement qui déborde...

O.A. : ... le cours habituel des choses, oui. Plusieurs recherches démontrent combien la préparation à un événement perturbateur est importante. Par exemple, si un patient s'est préparé psychologiquement pour une intervention chirurgicale, il récupère plus rapidement que s'il a banalisé l'événement. Cette observation s'applique aux traumatismes qui, par définition, surviennent sans préparation.

Je suis d'avis que l'approche du clinicien travaillant avec des victimes de traumatismes est la même qu'avec n'importe quel autre patient d'une pratique générale, à ceci près qu'il doit tenir compte du contexte très particulier de la consultation. En effet, ce type de patient dit: «Il m'est arrivé quelque chose qui m'a déstabilisé, qui m'a amené proche du précipice de la désorganisation psychique. Et je n'ai rien demandé à personne, moi. Je n'ai jamais voulu venir consulter un psychologue». Il n'y a pas de demande intérieure, elle est plutôt situationnelle. Il faut prendre ce fait en considération quand on travaille avec les victimes de traumatismes ponctuels.

T.N.: Oui. Mais il faut qu'ils soient vus?

O.A. : En général les victimes ne consultent pas. Toutes les recherches l'affirment. Il faut aller vers les victimes. C'est pour cette raison que le debriefing de groupe présente des avantages. Il contient aussi des inconvénients, et on peut en parler, mais l'avantage du debriefing consiste à rejoindre les victimes. Après le déluge au Saguenay, par exemple, des groupes de cliniciens sont allés dans les centres, dans les refuges, et ont expliqué aux gens : «On va parler de ce qu' on vit quand une telle épreuve survient pour vous aider à passer à travers, on va vous expliquer le fonctionnement émotionnel normal après une épreuve de ce genre. » Il faut être un peu proactif. Si on attend que les gens consultent, il n'en viendra pas beaucoup et le risque de stress post-traumatique chronique ou à survenue différée augmente. 
C'est sûr que les gens présentant une fragilité psychique sont plus à risque lors d'un événement traumatisant. Ils sont habituellement pris en charge par le système de santé. Mais comment aider le citoyen moyen victime d'un tel événement?

T.N.: Oui. Mais j'imagine que, même sans être dans un état de fragilité faisant qu'on est déjà suivi, les personnes vivant des situations d'inquiétude situationnelle telle une séparation, une difficulté familiale avec ses enfants, vont avoir plus de difficultés à gérer l'événement.

O.A.: Oui. Il existe des facteurs de vulnérabilité pré-traumatiques, les antécédents psychiatriques, comme on l'a dit, les traumatismes antérieurs non intégrés etc. il y a aussi des facteurs reliés au moment de la vie au cours duquel le traumatisme survient. Si quelqu'un est en processus de séparation, il est plus vulnérable. Mais il peut exister des facteurs plus anodins. Si mes trois enfants ont la grippe et que je dors très peu depuis trois jours, je serai plus vulnérable. Les facteurs pré-traumatiques interviennent sans que les victimes en soient conscientes dans un premier temps. Le travail clinique leur permet d'en prendre conscience. Il y a plusieurs méandres dans le traitement des réactions de stress post-traumatiques; on ne peut pas travailler uniquement sur le seul événement déclencheur, même si on doit lui accorder la plus grande importance dans un premier temps.

T.N. : Oui. J'imagine que les personnes seules, par exemple, un immigrant qui n'a pas de famille ici ou...

O.A.: Il s'agit là de facteurs post-événement. Ça comprend le degré de support, la gestion de la crise, l'attitude des patrons, etc. Par exemple, quand un patron n'est pas sur place au moment d'un hold-up dans une banque, on lui téléphone pour l'informer de ce qui vient de se passer dans sa succursale. S'il ne revient pas rencontrer ses employés et qu'il se contente de répondre: «Comptez ce qui reste dans la caisse et on verra demain». Pour certains employés, cette conduite inappropriée de la part du patron sera un traumatisme supplémentaire. Dans un moment de menace à la vie, l'autorité devient très importante, papa, maman... L'autorité qui protège. Les patrons sont vus comme des parents protecteurs ou non protecteurs. D'autres facteurs existent aussi. Par exemple, si les services d'aide n'arrivent pas assez rapidement sur les lieux. Si on ne voit pas à la sécurité physique des personnes et qu'on ne leur donne pas l'information dont ils ont besoin...

T.N. : Et que penser aussi de la meute des médias qui accourent pour interroger les victimes? Est-ce que ça ne fait pas partie aussi de facteurs...

O.A. : ... Post-traumatiques (rires).

T.N. : ... Aliénants?

O.A.: Je ne m'aventurerai pas sur ce sujet. Quand j'ai commencé à travailler en post-traumatique, les médias n'étaient pas très présents lors des Incidents critiques. Maintenant, ils sont très intrusifs. Et, bien sûr, certaines victimes se font prendre au piège de la réparation narcissique en répondant aux questions des journalistes. Mais cela ne constitue pas un facteur thérapeutique. Cela n'aide en rien.

T.N.: Parce que j'imagine que les victimes ont besoin de se retrouver dans une atmosphère de calme. 
O.A.: Oui. Les mesures à prendre après un sinistre contiennent cet élément: la protection immédiate des victimes contre les media. Il faut leur permettre de se retrouver entre elles, qu'elles puissent parler ou ne pas parler des événements, mais entre elles. Les étudiants de Concordia en septembre dernier ont compris cela quand ils ont organisé des lieux de rassemblement sécuritaires pour les étudiants du Collège Dawson. Les victimes sont dans un vécu trop intense et elles n’ont pas encore la distance nécessaire pour en témoigner publiquement. Je me souviens quand nous, les professionnels, sommes arrivés à Polytechnique en 1989, pour offrir des services psychologiques, les gens se regroupaient spontanément les uns avec les autres, par classes. Les professeurs préféraient être avec les étudiants avec lesquels ils se trouvaient au moment de la tuerie plutôt qu'avec leurs collègues. Il fallait respecter cette réaction, parce que entre eux...

T.N.: Ressouder cette intimité comme mesure réparatrice.

O.A. : Oui. Je dirais aussi que le groupe exerce une fonction contenante, telle que Bion l'a définie. Un traumatisme soulève un vécu trop intense pour être symbolisé sur le champ. En attendant d'être capables de faire cette démarche de symbolisation, qui se fait dans le temps et non sur le coup, les victimes ont souvent besoin d'alter ego et de support. Que ce soit leur groupe d'appartenance, à l'école ou au travail, que ce soit un ami ou le thérapeute, d'autres personnes exercent une fonction contenante. Il faut que quelqu'un «contienne» cette intensité émotionnelle. Les proches ne sont pas toujours capables de le faire. Cette fonction contenante peut s'intégrer dans la gestion des services psychologiques offerts aux victimes sans nécessairement l'identifier comme telle. En situation de crise, les psychologues ont souvent pu jouer un rôle conseil auprès des gestionnaires pour que cela soit fait d'une façon ou d'une autre.

T.N.: Oui. C'est un moyen de réparation naturel.

O.A. : Effectivement, la réparation va commencer, puisque les gens vont commencer à se parler entre eux : «Ah! toi aussi c'est comme ça, puis, mon dieu, je pensais qu'il y avait juste moi qui vivais cela comme ça. Puis...» Alors s'amorce le travail qui permet d'assumer le deuil, la perte de l'innocence, la perte des illusions voulant que «si on est une bonne personne qui fait son travail correctement, de telles catastrophes ne peuvent pas nous arriver». Ce qui n'est pas la réalité bien sûr.

T.N.: Oui.

O.A.: Je reviens aux différentes étapes de la réaction traumatique, toujours selon Horowitz. La première étape : l'état de choc, la deuxième étape : l'expression des émotions intenses (l'intrusion ou l'évitement) et la troisième étape où commence le processus de deuil et d'intégration du traumatisme dans l'histoire du sujet. Nous sommes dans le travail thérapeutique: nommer, représenter ce qui s'est passé, construire son histoire personnelle avec les faits vécus et l'apaisement émotionnel qui s'ensuit.

Les gens qui ne rendent pas à la troisième étape tombent dans la pathologie. Les gens se réparent de toutes sortes de façons, en prenant pied dans leur réalité ou dans leur réseau ou avec les personnes-ressources à leur disposition. 
T.N. : Avec les dangers aussi de réparer... Je pense, entre autres, à un client qui s'était mis à trop boire, ce n'était pas là une réparation efficace.

O.A.: Oui, certaines personnes adoptent des comportements qui masquent et aggravent la détresse morale. C'est après la période des émotions intenses qu'apparaissent ce qu'on appelle les symptômes, qu'ils soient physiques, cognitifs, émotifs ou comportementaux...

T.N.: Oui. Et les particularités de chacun.

O.A. : Et les particularités. Il y en a qui somatisent, d'autres qui se libèrent par des comportements inappropriés, comme de trop boire, de faire de la vitesse sur l'autoroute, etc. Tout cela doit être identifié et nommé.

Un exemple sympathique. Un groupe de concierges, avec qui je travaillais, à l'École Polytechnique, n'avaient pas grand-chose à dire sur ce qu'eux-mêmes ressentaient après la tuerie du 6 décembre 89 : «Bien oui, mais c'est arrivé, qu'estce que vous voulez qu'on fasse ? On est bien obligés d'y faire face. » «Bon c'est comme ça, c'est comme ça.» Ils étaient incapables de parler d'eux-mêmes, de ce qu'ils vivaient. Soudain, l'un d'eux dit: «Oui, moi j'ai quelque chose à dire. Moi, j'ai toujours ri des femmes, parce qu'elles se plaignent toujours d'avoir les pieds froids. Mais depuis que c'est arrivé ça, à Polytechnique, moi j'ai toujours les pieds froids et les mains moites. Pouvez-vous m'expliquer ça ?»Cela nous a permis d'ouvrir sur les sensations et les réactions liées à l'angoisse. Ce concierge, qui a eu le courage de parler de ses pieds froids, a pris conscience pour la première fois de sa vie que c'était peut-être là une façon d'être perturbé, déstabilisé, d'avoir peur pour ses enfants. Il a permis à ses collègues de s'ouvrir par la suite: «Non, moi ce n'est pas comme ça, moi, je bois ou j'ai envie de pleurer tout le temps. Mais ça, il ne faudrait pas que le boss entende ça.... » Les membres du groupe se sont mis à décrire tout ce qui leur arrivait, tout ce qu'ils vivaient depuis le 6 décembre. Et ce qu'ils avaient vécu pendant l'événement et ce qu'ils vivent depuis. Un debriefing, c'est le lieu d'acceptation des multiples différences dans le vécu de plusieurs êtres humains par rapport à un même événement traumatique.

Je voudrais souligner ici l'importance, dans les interventions de groupe, de ne pas laisser celui-ci se polariser autour de deux réactions. Par exemple: une personne pleure sans pouvoir s'arrêter et l'autre lui dit: «Bien voyons... Arrête ça, il faut se contrôler.». Il ne faut pas laisser ces deux personnes polariser la discussion du groupe autour de leur réaction antinomique. On doit donner l'espace de parole à la variété des réactions, émotions, symptômes. Il ne s'agit pas de reconstruire les faits mais d'exprimer des vécus différents, aussi vrais les uns que les autres. Ainsi, même ceux qui ne parlent pas se sentent impliqués, ils peuvent s'identifier à ce que d'autres expriment. Quand des critiques s'élèvent contre le debriefing, elles font souvent référence au fait que certaines victimes n'ont pu trouver d'espace de parole ou ne se sont pas reconnues dans les propos du groupe qui se sont centrés trop exclusivement sur certains vécus trop envahissants pour quelques membres du groupe. Et certaines personnes en sont perturbées. Il faut donc être très attentif à l'intensité émotionnelle. Qu'est-ce que ce groupe peut 
tolérer? Être capable d'un mouvement de va et vient dans le groupe entre l'expression de la détresse ou de la colère et une distance ou une défense ou une réserve par rapport à ces mêmes émotions.

T.N.: En même temps qu'on ne force pas les personnes à prendre la parole ?

O.A. : Non, je pense qu'il ne faut pas obliger les gens à parler, ni même à être dans un groupe.

T.N. : Non. J'imagine que le groupe doit présenter une certaine homogénéité pour qu'on puisse l'aider dans le cadre d'une rencontre d'une durée de deux à trois heures.

O.A. : Il existe différentes théories à ce sujet, mais, selon moi, il faut respecter les unités naturelles. Par exemple, si l'événement traumatique est survenu dans une classe, celle-ci devient l'unité naturelle. Il faut aussi regrouper les individus par niveau hiérarchique. On ne met pas ensemble le grand patron, le patron et les employés, parce qu'alors, personne ne dira devant son patron: «Je fais des cauchemars toutes les nuits, et je ne travaille pas bien le lendemain matin. » Il faut une certaine homogénéité et respecter les unités naturelles. Je me souviens d'un centre pour personnes âgées où s'était déclaré un incendie. Les employés nous ont demandé que les groupes soient formés par unité de travail ce jour-là et non par profession : infirmières, préposés, etc.

Un souvenir concernant cet événement. Vous ayez parlé des médias... Ce qui avait été rapporté dans les médias, à propos de cet incendie, c'était que tout s'était parfaitement bien déroulé, il n'y avait eu aucun blessé, aucun mort, les cent bénéficiaires avaient été évacuées de l'édifice sans problème. Or, le travail dans les groupes de debriefing avait révélé tout l'aspect traumatique vécu par les employés.

Quand il y a un feu dans un établissement, il faut évacuer les gens par les escaliers et non par les ascenseurs. Or, tous les bénéficiaires sont des gens qui ont de la difficulté à marcher. Il faut alors convaincre madame une telle de prendre l'escalier et non pas l'ascenseur. Il faut lui expliquer qu'il faut qu'elle sorte tout de suite. Et que, non, on n'ira pas jusqu'à son lit pour qu'elle aille chercher son chapelet, son chapeau ou ses gants. Une autre difficulté vécue comme traumatique par les employés: le choix du bénéficiaire à évacuer en premier quand deux personnes sont ensemble, parce que : «Rien ne m'assurait que je serais capable de revenir chercher l'autre». L'exemple de cet incendie illustre bien le caractère subjectif de ce qui affecte les gens. Oui, il y a eu un feu, tout le monde a parlé de l'incendie. Mais c'est la façon dont il a été vécu par chacun qui le rend traumatique ou non et c'est cette perception subjective qui doit faire l'objet du travail clinique. T.N.: Quand on travaille avec des groupes, par exemple, des groupes d'élèves, il est important que les parents soient près d'eux après l'épreuve. J'imagine que vous leur donnez aussi un temps d'information pour les aider à entourer adéquatement leurs enfants...

O.A.: Oui, des informations générales et des consignes sont communiquées aux parents et aux familles en général, s'il y a lieu: respecter, ne pas juger, ne pas donner de conseils, ne pas dire: «Ne t'en fais pas, ça va passer», «N'y pense 
pas », accueillir les victimes dans ce qu'ils ont à dire, mais ne pas les forcer à parler.

T.N.: J'imagine que des enjeux personnels peuvent amener des gens à dédramatiser une situation, ce qui est une autre façon de...

O.A. : ... Se protéger de la souffrance morale.

T.N.: Oui. Mais, cela constitue pour la victime un désaveu de sa souffrance.

O.A.: Les victimes vivront inévitablement de l'incompréhension et la solitude qui en résulte. Quand elles souffrent, les gens autour d'elles se protègent comme ils peuvent de cette souffrance. Tous ne peuvent pas l'accepter, l'entendre, l'accueillir. Certains proches non seulement s'en défendent en se sauvant, mais aussi en attaquant et les victimes se sentent alors très seules. Et c'est inévitable. Cela fait partie des difficultés des victimes. Elles parlent toutes de la solitude morale.

T.N.: C'est vrai; si on n'a pas été dans l'événement, il est difficile de se le représenter, puisqu'on parle de l'éclatement du sens.

O.A.: Oui, tout à fait.

T.N.: C'est de la mort dont il est question, de la mort de la pensée, à tout le moins.

O.A.: Oui, et de la perte du pouvoir personnel. Être victime du pouvoir ou de la violence de l'autre, de l'indifférence de la nature ou de... C'est se retrouver dans une situation où, pendant un certain temps, je n'ai plus aucun pouvoir sur moimême. Il s'agit donc de survivre à un état d'impuissance personnelle. Pour aider les gens, il faut les amener à retrouver ce pouvoir personnel d'une façon ou d'une autre. Bien sûr, en parlant, en nommant, mais par d'autres moyens aussi ; cela peut être en bougeant, en faisant des activités qui font du sens pour eux. L'entourage peut avoir de la difficulté à accepter la souffrance morale de la victime, parce qu'elle est contagieuse. Si je vous parle du sentiment d'horreur ou de dégoût que j'ai vécu quand, durant un incendie, quelqu'un a brûlé devant moi, vous allez vous aussi le ressentir. Et si vous êtes incapable de le porter, vous allez vous en défendre et vous ne m'aiderez pas à le porter. Et ce sentiment d'impuissance est très difficile à vivre. Les gens s'en défendent de n'importe quelle façon et la culpabilité est une des façons de s'en défendre. Il est souvent plus facile de se sentir coupable dans un premier temps. C'est pourquoi on ne doit pas priver les victimes de leur sentiment de culpabilité. Il faut leur dire que, non, elles ne sont pas responsables de ce qui est arrivé, mais la culpabilité peut être une façon de reprendre pied: «Si j'avais fait ça, il ne serait pas arrivé telle chose. Ou si je m'étais caché sous le pupitre, je n'aurais pas vu ce qui m'a tellement bouleversé. » C'est une façon de retrouver son pouvoir que de se livrer à de telles pensées. On négocie avec la réalité.

T.N.: Oui. Vous avez surtout travaillé avec des victimes adultes?

O.A. : Oui, presque uniquement avec des victimes adultes.

T.N.: Je peux me représenter comment le travail avec des enfants en situation de traumatismes aussi sévères doit solliciter de façon... Incroyable.

O.A.: Oui. Mais les enfants sont plus spontanés et il leur est peut-être plus facile de se livrer à une activité symbolique. Les adultes sont plus réservés, plus conscients du jugement des autres. Je n'ai pas encore mentionné les activités 
symboliques; elles sont aussi un instrument thérapeutique très important. Quand décède, dans l'exercice de ses fonctions, un membre d'un corps de métier portant un uniforme de travail qui l'identifie : un policier, un pompier, un camionneur, par exemple, il arrive souvent que ses collègues mettent leur uniforme, nettoient leurs camions, organisent une parade lors des funérailles. Ce geste symbolique semble les aider à réparer ou à lutter contre l'impuissance vécue lors de l'accident mortel. Leur collègue est décédé et ils lui rendent un hommage funéraire. C'est important. Dans l'intervention de conseil auprès des gestionnaires, le professionnel fait penser à organiser ce genre d'activités symboliques réparatrices.

T.N. : Les événements collectifs servent à ne pas rester dans la solitude, à ce que les choses soient nommées, reconnues. Comme pour les survivants des camps de concentration...

O.A. : C'est ça. Tout à fait.

T.N. : Le devoir de mémoire, finalement. Et ne pas rester seul avec un événement qui nous a totalement pris la tête, comme on dit.

O.A. : Oui. Qui nous a dépossédés de nous-mêmes.

T.N. : Dans les divers événements où vous avez eu à intervenir, est-ce que certains sont restés pour vous des expériences extrêmement marquantes, prégnantes?

O.A.: Ah, c'est sûr que c'est la tuerie de Polytechnique en 1989. C'est l'événement le plus important pour lequel j'ai travaillé dans ma carrière et celui qui m'a le plus troublée. On était un grand nombre de psychologues et d'intervenants, toute une logistique supportait les services psychologiques. C'était le premier événement de ce genre et il y avait aussi l'intensité et l'ampleur de ce que les gens vivaient. La population affectée formait comme des cercles concentriques. On parlait beaucoup des quatorze jeunes filles décédées. Il y avait des blessés physiques. Mais il y avait aussi des centaines et des centaines de personnes blessées psychologiquement. Des étudiants, des employés sur place ou dont c'était le lieu de travail, les familles, les amis... En fait, le Québec tout entier était en état de choc. On pouvait dessiner des cercles de victimes directes ou indirectes presque à l'infini, tant le nombre de personnes affectées par l'événement était immense. Et dans l'après-coup, plusieurs étudiants de Polytechnique ont été harcelés parce qu'ils n'avaient pas défendu les filles, alors que personne, comme je le disais plus tôt, ne savait ce qui se passait au moment où la tuerie se déroulait. Et, surtout, qu'est-ce qu'un étudiant de dix-neuf ans a de plus qu'une jeune fille de dix-neuf ans pour se défendre devant une carabine tronçonnée dans notre société ? Ces commentaires ont ajouté au traumatisme des étudiants.

T.N. : Oui. Re-traumatisant.

O.A.: Oui. Alors Polytechnique fut l'expérience la plus marquante de ma carrière en post-traumatique. Ce fut aussi l'événement le plus riche au niveau de la collaboration. Il y a un temps de grâce après un événement traumatique où l'on voit le meilleur des gens et des professionnels impliqués. Des gestes sont posés qui coupent le souffle. Les gens sont tellement gentils, adéquats, compatissants, généreux les uns avec les autres. Mais ce temps de grâce ne dure qu'un temps. 
T.N.: La vie reprend ses droits.

O.A.: La vie reprend ses droits. Et le temps de grâce prend fin. Ce premier moment de compassion aide beaucoup les victimes ainsi que les intervenants.

T.N.: Qu'est-ce qui vous a amenée à laisser?

O.A.: La fatigue. C'est de la médecine de guerre, d'abord parce que les événements traumatiques surviennent sans prévenir. Il faut gérer son horaire pour laisser de la place à l'imprévu, tout en ne sachant ni le nombre, ni la fréquence des événements : y en aura-t-il trois par semaine ou un par trois mois? Un auteur américain, Figley, parle de stress traumatique secondaire qui menace les thérapeutes. Et ça existe. J'avançais en âge et c'était le temps de laisser la place aux jeunes. J'ai cessé de faire de l'intervention dans un premier temps et j'ai continué à faire de la formation encore pendant quelques années.

T.N.: Qu'est-ce qui a été prévu pour l'épuisement professionnel dans votre métier? Parce qu'on peut parler ici de traumatisme vicariant...

O.A. : Je n'ai jamais exercé ce type de pratique à plein temps. J'ai toujours intégré cette activité à un autre travail. Il faut faire attention à soi. Je n'encourage d'ailleurs pas les jeunes psychologues à pratiquer le debriefing de façon répétitive. Même si on a beaucoup d'énergie et de bonne volonté, ce genre de travail exige un rythme d'intervention raisonnable, qui nous respecte nous-mêmes. On ne peut pas non plus, à mon avis, faire une intervention de debriefing de groupe le matin et recevoir cinq clients l'après-midi. On n'a pas la neutralité ni la disponibilité émotionnelle après une séance de debriefing.

T.N.: Si on regardait les théories qui soutiennent ce travail? Des controverses existent à propos du modèle médical américain proposant sept étapes dans l'intervention post-traumatique. On commence par la narration des faits, on passe ensuite aux réactions des gens, qu'est-ce qu'ils ont pensé. Pour en arriver petit à petit aux émotions, mais toujours en dernier. À l'opposé, le modèle européen suggère que le traumatisme induise une perte du sens de la vie, du sens de la parole de l'être humain. Il faut donc récupérer cette parole par la mise en mots, la mise en circulation de la parole. Et cette parole est accueillie comme elle vient, qu'elle parle d'émotions ou de narration de faits ou de réactions physiques. Qu'elle est votre position par rapport à ces modèles?

O.A. : J'ai commencé à travailler avant la mise au point de ces modèles. J'ai utilisé les théories psychologiques et psychanalytiques comme des paires de lunettes pour mieux voir la réalité de ce qui se passait. Mais quand on parle de debriefing de groupe, on parle d'interventions de crise, d'interventions court terme dans le «ici et maintenant». Les théories psychanalytiques sont utiles pour la lecture de la dynamique des groupes et des individus, la lecture des enjeux entre la vie et la mort, l'identification des mécanismes de défense, des processus de régression, etc. Elles servent à la réflexion sur ce qui se passe. Quant aux interventions, elles se rattachent davantage au modèle humaniste et à la dynamique des groupes développée à l'intérieur de ce courant théorique. L'attitude du thérapeute tout en gardant son empathie et sa neutralité sera cependant plus directive. L'objectif n'est 
pas d'ouvrir mais de fermer. Concernant les modèles auxquels vous faites référence, je serais plutôt du côté du modèle européen. Le modèle américain s'applique mais pas nécessairement dans l'ordre.

T.N. : Vous ne vous inscrivez pas non plus dans le courant théorique behavioral? O.A. : Non.

T.N.: Les modèles médicaux s'inscrivent souvent dans ces théories.

O.A. : Oui, c'est ça. Et il est vrai que le courant behavioriste chapeaute maintenant l'intervention post-traumatique en général. Ce sont les firmes de Programmes d'aide aux employés qui offrent ce service avec des modèles d'intervention précis. T.N.: Quand vous regardez vos vingt ans de pratique en post-traumatique, quel bilan en faites-vous? Avez-vous l'impression d'avoir été changée par le fait de travailler régulièrement avec des gens qui ont vécu des expériences limites ?

O.A.: Oui, vous avez le terme juste. C'est sûr que cet aspect de mon travail clinique a eu un grand impact sur moi. J'ai l'impression d'avoir appris à naviguer en haute mer. Je suis devenue plus consciente des différences entre la psychothérapie individuelle et les interventions à court terme. Les objectifs ne sont pas les mêmes. D'abord le post-traumatique exige de travailler dans le désordre, alors que le travail en pratique privée est et doit être beaucoup plus ordonné du moins dans ses paramètres. Après un événement traumatique, on travaille dans le désordre et en cela, ça ressemble beaucoup à la vie. Le thérapeute ou l'animateur du groupe doit être capable d'écouter, de contenir, de garder la communication fluide, d'articuler une pensée rapidement, de nommer ce qui est exprimé avec difficulté, de contenir, de supporter, de ne pas juger, de vulgariser. D'ailleurs, vous l'avez probablement remarqué, j'utilise peu les termes théoriques ou techniques...

T.N.: Oui.

O.A. : C'est parce que j'ai vraiment voulu parler simplement pour que les victimes comprennent sans faire d'effort intellectuel, car la concentration n'est pas très grande après un incident traumatique. Il faut donc dire les choses d'une façon concise, claire, qui les rejoigne, en utilisant leur vocabulaire. Cette exigence m'a beaucoup fait travailler. Et les gens sont très reconnaissants que tout ce tumulte prenne un sens pour eux. L'intervention post-traumatique est un des domaines cliniques où le psychologue constate assez rapidement un certain résultat de son travail. Pour les cliniciens au tempérament actif, il existe une satisfaction à pouvoir voir rapidement les effets de ses interventions. Mais il y a l'usure de la rapidité d'intervention et de l'immersion dans l'intensité émotionnelle. Je redis ici que ce sont des interventions psycho-éducationnelles et non psychothérapeutiques.

J'ai aussi aimé pratiquer l'intervention conseil auprès des gestionnaires et des organismes. C'était une occasion d'enseigner le fonctionnement psychologique à des gens qui dirigent des dizaines d'employés, et d'avoir ainsi un effet multiplicateur.

T.N. : Et l'ouverture était là ?

O.A. : Pas toujours.

T.N.: Mais sur vingt ans, avez-vous vu quand même des changements dans les grandes organisations? 
O.A. : J'ai vu une courbe se déplacer. Au début, les gens étaient très réticents. Je me souviens, au moment où j'ai décidé de faire le tour des directeurs de Caisses Populaires pour leur expliquer ce que vivaient leurs employés quand il y avait un hold-up dans une caisse ou une succursale bancaire. Obtenir une invitation pour parler des hold-up, c'était à peu près impossible parce qu'il ne fallait pas «tenter le diable», c'est-à-dire parler de hold-up. Puis, s'est produite une petite ouverture et les directeurs m'accordaient vingt minutes. Puis, une demi-heure. Quelques années plus tard, on faisait des formations de trois heures aux gestionnaires... Il y a eu une ouverture à la fin des années 1980, après la tragédie de Polytechnique. À ce moment, les entreprises et les institutions où peuvent survenir des accidents de travail, les banques et les caisses, les compagnies de transport, les compagnies ferroviaires dont les conducteurs de train, par exemple, peuvent voir quelqu'un se jeter sur la voie et eux savent qu'ils ne pourront pas arrêter le train, plusieurs de ces organisations se sont intéressées aux services psychologiques posttraumatiques. Il est donc survenu une grande ouverture, qui s'est ensuite un peu refermée. Comme un retour du balancier: "Ça suffit, on va arrêter de parler de ça et on va plutôt parler de production, de qualité, de rendement...»

T.N. : Oui.

O.A.: Je ne sais plus, depuis le début des années 2000 , ce qui se passe exactement en post-traumatique, alors il faudrait demander à ceux qui sont encore actifs où en sont rendues les choses. Mais, dans le courant de ma carrière, $\mathrm{j}$ ' ai vu une ouverture se produire sur plusieurs années, puis une fermeture se faire sur plusieurs autres. T.N. : Oui. On va terminer ici. Là-dessus je vous remercie de votre témoignage. Je vous souhaite une belle fin de carrière. Et encore beaucoup de plaisir dans votre travail

O.A.: Merci beaucoup.

odette arsenault

500-1259 berri montréal

Qc H2L 4C7

thérèse nadeau

39 , gouin ouest montréal

Qc H1X 3X8

nadeaut@videotron.ca 